\title{
CRITICALLY $n$-CONNECTED GRAPHS
}

\author{
GARY CHARTRAND, ${ }^{1}$ AGNIS KAUGARS AND DON R. LICK ${ }^{1,2}$
}

\begin{abstract}
The following result is proved. Every $n$-connected graph contains either a vertex whose removal results in a graph which is also $n$-connected or a vertex of degree less than $(3 n-1) / 2$.
\end{abstract}

Introduction. A graph $G$ is said to be n-connected if the removal of fewer than $n$ vertices from $G$ neither disconnects it nor reduces it to the trivial graph consisting of a single vertex. The maximum value of $n$ for which a graph $G$ is $n$-connected is called its connectivity and is denoted by $\kappa(G)$. The minimum degree of $G$ is designated by $\delta(G)$; the inequality $\kappa(G) \leqq \delta(G)$ is well known.

A graph $G$ is said to be critically $n$-connected if $\kappa(G)=n$ and $\kappa(G-v)=$ $n-1$ for each vertex $v$ of $G$. Analogously, a graph $G$ is minimally $n$ connected if $\kappa(G)=n$ and for each edge $e$ of $G, \kappa(G-e)=n-1$. The object of this article is to present a necessary condition for a graph to be critically $n$-connected and to discuss related topics.

Since 1-connected graphs are the nontrivial connected graphs and since every nontrivial connected graph $G$ has at least two vertices $u$ and $v$ such

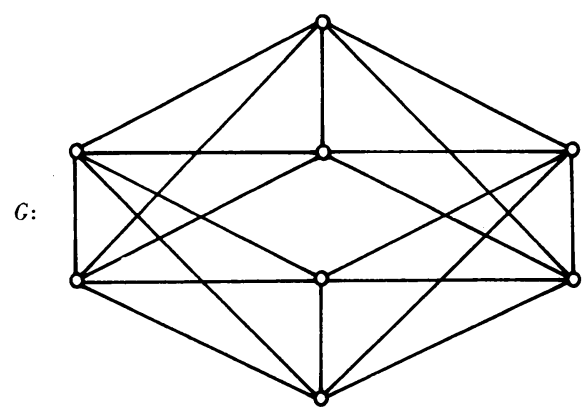

Figure 1

Received by the editors August 8, 1968 and, in revised form, April 17, 1969.

AMS 1969 subject classifications. Primary 0540.

Key words and phrases. Graph, connectivity, critically $n$-connected.

${ }^{1}$ Research supported in part by grant GP-9435 from the National Science Foundation.

${ }^{2}$ Research supported in part by a Faculty Research Fellowship from Western Michigan University.

(c) American Mathematical Society 1972 
that each of $G-u$ and $G-v$ is connected, it follows that the only critically 1 -connected graph is the complete graph of order two. It is also easily observed that a graph is minimally 1-connected if and only if it is a nontrivial tree; thus if $G$ is a graph which is either critically 1-connected or minimally 1-connected, then $\delta(G)=1$. Dirac [2] and Plummer [7] have shown that if $G$ is minimally 2 -connected then $\delta(G)=2$. Recently, Halin [4] extended this result so that if $G$ is a minimally $n$-connected graph, $n \geqq 1$, then $\delta(G)=n$. It was shown in [5] that every critically 2-connected graph has minimum degree 2 . The graph in Fig. 1 shows that no theorem on critically $n$-connected graphs analogous to Halin's thzorem on minimally $n$-connected graphs is possible. The graph $G$ of Fig. 1 is critically 4-connected but $\delta(G)=5$.

We shall prove that every critically $n$-connected graph, $n \geqq 2$, has a vertex of degree less than $(3 n-1) / 2$ and that the number $(3 n-1) / 2$ cannot be improved.

Preliminaries. Before proceeding further, it is convenient to give a few definitions and establish some notation. All terms not defined here may be found in Harary [3].

If $U$ is a nonempty subset of the vertex set $V(G)$ of $G$, then the subgraph $H$ induced by $U$, written $H=\langle U\rangle$, is the subgraph whose vertex set is $U$ and where two vertices are adjacent if and only if these vertices are adjacent in $G$. A set $S$ of vertices of $G$ is called a cut set of $G$ if the (induced) subgraph $G-S=\langle V(G)-S\rangle$ is disconnected; $S$ is an $n$-cut set if $|S|=n$. Two paths of $G$ are said to be disjoint if they have no vertices in common except possibly end vertices.

Two special classes of graphs which we shall encounter are the complete graphs and the complete bipartite graphs. The complete graph $K_{p}$ has $p$ vertices every two of which are adjacent. The complete bipartite graph $K(m, n)$ has its vertex set $V$ partitioned into two subsets $V_{1}$ and $V_{2}$, where $\left|V_{1}\right|=m$ and $\left|V_{2}\right|=n$, such that two vertices $u$ and $v$ are adjacent if and only if $u \in V_{i}$ and $v \in V_{j}, i \neq j$.

The concepts of "critically $n$-connected" and "minimally $n$-connected" are independent in the sense that neither property implies the other. For
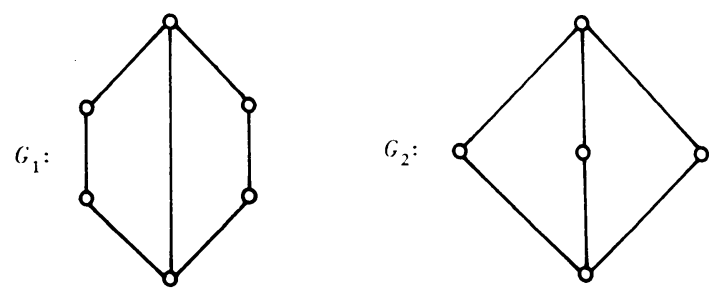

FIGURE 2 
example, the graph $G_{1}$ of Fig. 2 is critically 2 -connected but not minimally 2-connected while $G_{2}=K(2,3)$ is minimally 2-connected and not critically 2-connected. In general, the graph $K(n, n+1)$ is minimally $n$-connected but not critically $n$-connected. For $n \geqq 3$, the graph obtained by adding an extra edge to $K(n, n)$ is critically $n$-connected but not minimally $n$-connected.

We note that it is rarely easy to ascertain whether a given graph is critically $n$-connected for some $n$. Despite this fact, such graphs are quite numerous; indeed if $G$ is $n$-connected and $G^{\prime}$ is a subgraph of $G$ containing the minimum number of vertices such that $G^{\prime}$ is $n$-connected, then $G$ is critically $n$-connected.

A necessary condition for critically $n$-connected graphs. We now present the main result of this article.

THEOREM. If $G$ is a critically $n$-connected graph, $n \geqq 2$, then $\delta(G)<$ $(3 n-1) / 2$ and the number $(3 n-1) / 2$ cannot be improved.

Proof. Suppose the theorem to be false so that there exists a graph $G$ of order $p$ having $\kappa(G)=n$ and $\delta(G) \geqq(3 n-1) / 2$ such that for every $v \in V(G), \kappa(G-v)=n-1$. We note that since $\delta(G) \geqq(3 n-1) / 2, G$ is not complete. This implies that every vertex of $G$ belongs to some $n$-cut set of $G$.

Among all $n$-cut sets $S^{\prime}$ of $G$, let $S$ be one such that $G-S$ contains a component $G_{1}$ of smallest order; denote the order of $G_{1}$ by $m$. Furthermore, let $G_{2}=G-S-V\left(G_{1}\right)$.

Let $v \in V\left(G_{1}\right)$ and $u \in V\left(G_{2}\right)$. By a result of Whitney [8] there exist $n$ disjoint $u-v$ paths in $G$; necessarily, each such path contains precisely one vertex of $S$. Hence there exist $n$ disjoint paths joining $u$ and $S$ (and also $v$ and $S$ ).

Let $w \in V\left(G_{1}\right)$, and let $S^{*}$ be an $n$-cut set of $G$ containing $w$. Define $G^{*}=G-S^{*}$ and, furthermore, let $V_{1}=V\left(G_{1}\right) \cap S^{*}, V_{2}=V\left(G_{2}\right) \cap S^{*}$, and $V_{3}=S \cap S^{*}$, where $\left|V_{i}\right|=n_{i}, i=1,2,3$. We note that $n_{1}+n_{2}+n_{3}=n$ and $n_{1} \geqq 1$.

We now show that $n_{2} \geqq n_{1}$. If $S^{*} \supseteq V\left(G_{2}\right)$, then this is obvious. Assume therefore that $V\left(G_{2}\right)-V_{2} \neq \varnothing$. We have already noted that for each $u \in V\left(G_{2}\right)$, there exists in $G$ a set of $n$ disjoint paths joining $u$ and $S$. If $u \in V\left(G_{2}\right)-V_{2}$, then at least $n-n_{2}-n_{3}=n_{1}$ of these paths contain no vertices of $V_{2} \cup V_{3}$. In this case, denote the set of end vertices in $S$ of these $n_{1}$ (or more) paths by $R(u)$. Thus for each $u \in V\left(G_{2}\right)-V_{2}$, there exists a set $R(u) \subset S-V_{3}$ such that there are disjoint paths containing no elements of $V_{2} \cup V_{3}$ which join $u$ and $R(u)$ where $|R(u)| \geqq n_{1}$. If there exist vertices $u_{1}$, $u_{2} \in V\left(G_{2}\right)-V_{2}$ such that $R\left(u_{1}\right) \cap R\left(u_{2}\right)=\varnothing$, then $\left|S-V_{3}\right| \geqq 2 n_{1}$ so that $n-n_{3} \geqq 2 n_{1}$ and $n_{2} \geqq n_{1}$. Otherwise, let $R=\bigcup R(u)$, the union taken over all 
$u \in V\left(G_{2}\right)-V_{2}$, and let $G^{\prime}=\left\langle R \cup\left(V\left(G_{2}\right)-V_{2}\right)\right\rangle$. It is now easy to verify that every two vertices of $G^{\prime}$ are connected so that $G^{\prime}$ itself is connected. Hence $G^{\prime}$ is a subgraph of a component of $G^{*}$. Since the order of $G^{\prime}$ is at least $n_{1}+(p-m-n)-n_{2}$, there must be a component of $G^{*}$ of order at most $m+n_{2}-n_{1}$. Therefore, $m \leqq m+n_{2}-n_{1}$ so that $n_{2} \geqq n_{1}$. Thus in any case, $n_{2} \geqq n_{1}$.

The inequality $n_{2} \geqq n_{1}$ implies that $n_{1} \leqq n / 2$. We next verify that $V\left(G_{1}\right)-$ $V_{1} \neq \varnothing$ or, equivalently, that $n_{1}<m$. Assume that $n_{1}=m$ so that $V\left(G_{1}\right)=V_{1}$. Hence for each $v \in V\left(G_{1}\right)$,

$$
\operatorname{deg} v \leqq\left(n_{1}-1\right)+n \leqq(3 n-2) / 2
$$

which contradicts the fact that $\delta(G) \geqq(3 n-1) / 2$. We conclude therefore that $n_{1}<m$ and $V\left(G_{1}\right)-V_{1} \neq \varnothing$.

Let $F=\left\langle\left(V\left(G_{1}\right)-V_{1}\right) \cup\left(S-V_{3}\right)\right\rangle$. We show that $F$ is disconnected. Suppose, to the contrary, that $F$ is a connected subgraph of $G^{*}$. Since $G^{*}$ is not connected, $V\left(G_{2}\right)-V_{2} \neq \varnothing$. Because each $u \in V\left(G_{2}\right)-V_{2}$ is joined to $S-V_{3}$ by at least $n_{1}$ paths in $G^{*}$, it follows that $G^{*}$ is connected which is impossible. Thus $F$ is disconnected.

Denote the components of $F$ by $F_{t}, t=1,2, \cdots, k$, where $k \geqq 2$. Furthermore, for each $t=1,2, \cdots, k$, denote by $W_{t}$ the set of vertices of $F_{t}$ in $S$, where $\left|W_{t}\right|=s_{t}$. We note that each $W_{t} \neq \varnothing$; for otherwise there would exist a component of $F$ of order less than $m$ contained in $\left\langle V\left(G_{1}\right)-V_{1}\right\rangle$ which would also be a component of $G^{*}$.

We claim that precisely one of the subgraphs $F_{t}$ contains elements of $V\left(G_{1}\right)-V_{1}$. Assume this is not the case so that there are two subgraphs $F_{i}$ and $F_{j}, i \neq j$, containing elements of $V\left(G_{1}\right)-V_{1}$. Let $W_{i}^{\prime}=\bigcup W_{t}, t \neq i$, where $\left|W_{i}^{\prime}\right|=s_{i}^{\prime}$. Each of the sets $V_{1} \cup V_{3} \cup W_{i}$ and $V_{1} \cup V_{3} \cup W_{i}^{\prime}$ is a cut set of $G$, for in each case the removal of the set from $G$ produces a graph having a component contained in $\left\langle V\left(G_{1}\right)-V_{1}\right\rangle$. This implies that $n_{1}+n_{3}+s_{i} \geqq n$ and $n_{1}+n_{3}+s_{i}^{\prime} \geqq n$ so that $s_{i} \geqq n_{2}$ and $s_{i}^{\prime} \geqq n_{2}$. However, the equality $n_{1}+n_{2}+n_{3}=s_{i}+s_{i}^{\prime}+n_{3}=n$ together with the inequality $n_{2} \geqq n_{1}$ yield $s_{i}=s_{i}^{\prime}=n_{1}=n_{2}$. Therefore, $V_{1} \cup V_{3} \cup W_{i}$ is an $n$-cut set of $G$, but the graph $G-\left(V_{1} \cup V_{3} \cup W_{i}\right)$ has a component of order less than $m$. This produces a contradiction; hence exactly one of the subgraphs $F_{t}$ contains elements of $V\left(G_{1}\right)-V_{1}$. Let $F_{1}$ be the subgraph with this property.

Now $V_{1} \cup V_{3} \cup W_{1}$ is a cut set of $G$ so that $n_{1}+n_{3}+s_{1} \geqq n$ or $s_{1} \geqq n_{2}$. Let $G_{1}^{*}$ be a component of $G^{*}$ which contains vertices of $W_{1}^{\prime}$. If $V\left(G_{1}^{*}\right) \subseteq W_{1}^{\prime}$, then $s_{1}^{\prime} \geqq m$, but this implies that

$$
n=s_{1}+s_{1}^{\prime}+n_{3} \geqq n_{2}+m+n_{3}>n_{2}+n_{1}+n_{3}=n,
$$

which is impossible. Therefore, $G_{1}^{*}$ contains vertices of $V\left(G_{2}\right)-V_{2}$, which incidentally shows that $V\left(G_{2}\right)-V_{2} \neq \varnothing$. 
We show next that $V_{2} \cup V_{3} \cup W_{1}^{\prime}$ is a cut set of $G$. Suppose this is not so. Then $G^{\prime}=G-\left(V_{2} \cup V_{3} \cup W_{1}^{\prime}\right)$ is connected. Since $F_{1}$ is connected, the graph $G^{\prime \prime}=G^{\prime}-V_{1}$ is also connected. However, $G^{*}=\left\langle V\left(G^{\prime \prime}\right) \cup W_{1}^{\prime}\right\rangle$ is disconnected; therefore, $G^{*}$ has a component which is a subgraph of $\left\langle W_{1}^{\prime}\right\rangle$, but we have seen that every component of $G^{*}$ which contains elements of $W_{1}^{\prime}$ also contains elements of $V\left(G_{2}\right)-V_{2}$. Hence $G-\left(V_{2} \cup V_{3} \cup W_{1}^{\prime}\right)$ is disconnected so that $V_{2} \cup V_{3} \cup W_{1}^{\prime}$ is a cut set of $G$. This produces the inequality $n_{2}+n_{3}+s_{1}^{\prime} \geqq n$ or $s_{1}^{\prime} \geqq n_{1}$.

We now know that $s_{1}+s_{1}^{\prime}=n_{1}+n_{2}, s_{1} \geqq n_{2}$, and $s_{1}^{\prime} \geqq n_{1}$. From this we conclude that $s_{1}=n_{2}$ and $s_{1}^{\prime}=n_{1}$. Returning to the cut set $V_{1} \cup V_{3} \cup W_{1}$, we note that this is an $n$-cut set. However, $G-\left(V_{1} \cup V_{3} \cup W_{1}\right)$ contains a component of order less than $m$. This produces a contradiction, and the desired result follows.

Using the construction in [6], we show that the number $(3 n-1) / 2$ cannot be improved, i.e., for each positive integer $n$ and positive integer $m<(3 n-1) / 2$, there is a critically $n$-connected graph $G$ with $\delta(G)=m$. Before giving the construction, we define the join of two graphs. The join of two graphs $G_{1}$ and $G_{2}$, denoted by $G_{1}+G_{2}$, is the union $G_{1} \cup G_{2}$ of $G_{1}$ and $G_{2}$ together with all edges of the type $v_{1} v_{2}$ where $v_{i}$ is a vertex of $G_{i}$, $i=1,2$.

For $n \geqq 2 m+2$, define the collection $\left\{H_{n, m}\right\}$ of graphs as follows:

$$
\begin{aligned}
H_{n, m} & =2 K_{m+1}, & & \text { for } n=2 m+2, \\
& =K_{n-2 m-2}+2 K_{m+1}, & & \text { for } n>2 m+2 .
\end{aligned}
$$

It is easily seen that $H_{n, m}$ has order $n$ and $\delta\left(H_{n, m}\right)=n-m-2$. Using a result in [1], the equality $\kappa\left(H_{n, m}\right)=n-2 m-2$ follows.

For $n<m<(3 n-1) / 2$, define

$$
G_{n, m}=H_{n, m-n}+2 K_{m-n+1} \text {. }
$$

The graph $G$ given in Fig. 1 is $G_{4,5}$. From the information obtained about $H_{n, m}$, it follows that $\delta\left(G_{n, m}\right)=m$, and with the aid of the above-mentioned result in [1], $\kappa\left(G_{n, m}\right)=n$. Let $v$ be a vertex of $G_{n, m}$. If $v$ belongs to $H_{n, m-n}$, then the removal of $v$ and the remaining $n-1$ vertices of $H_{n, m-n}$ results in a disconnected graph; thus, $\kappa\left(G_{n, m}-v\right)=n-1$. If $v$ belongs to $2 K_{m-n+1}$, then the removal of the vertices of $2 K_{m-n+1}$ together with a $(3 n-2 m-2)$ cut set of $H_{n, m-n}$ gives a disconnected graph. Hence, here too we have $\kappa\left(G_{n, m}-v\right)=n-1$. The graph $G_{n, m}$ is therefore critically $n$-connected.

\section{REFERENCES}

1. G. Chartrand and F. Harary, Graphs with prescribed connectivities, Theory of Graphs (Proc. Colloq., Tihany, 1966), Academic Press, New York, 1968, pp. 61-63. MR 38 \#4346. 
2. G. A. Dirac, Minimally 2-connected graphs, J. Reine Angew. Math. 228 (1967), 204-216. MR 36 \#70.

3. F. Harary, Graph theory, Addison-Wesley, Reading, Mass., 1969. MR 41 \#1566.

4. R. Halin, A theorem on n-connected graphs, J. Combinatorial Theory 7 (1969), 150-154. MR 40 \#1297.

5. A. Kaugars, $A$ theorem on the removal of vertices from blocks, Senior Thesis, Kalamazoo College, Kalamazoo, Mich. 1968.

6. D. R. Lick, Connectivity preserving subgraphs, Math. Report \#1, Western Michigan University, Kalamazoo, Mich., 1968.

7. M. D. Plummer, On minimal blocks, Trans. Amer. Math. Soc. 134 (1968), 85-94. MR 37 \#3950.

8. H. Whitney, Congruent graphs and the connectivity of graphs, Amer. J. Math. 54 (1932), 150-168.

Department of Mathematics, Western Michigan University, Kalamazoo, MICHIGAN 49001

Department of Mathematics, Harvard University, Cambridge, Massachusetts 02138 